\title{
Ginsenoside Rh2 promotes nonamyloidgenic cleavage of amyloid precursor protein via a cholesterol-dependent pathway
}

\author{
J. Qiu ${ }^{1 *}$, W. Li ${ }^{2 *}$, S.H. Feng ${ }^{3}$, M. Wang ${ }^{3}$ and Z.Y. He ${ }^{1}$ \\ ${ }^{1}$ Department of Neurology, First Hospital of China Medical University, \\ Shenyang, China \\ ${ }^{2}$ Department of Neurology, General Hospital of Shenyang Military Command, \\ Shenyang, China \\ ${ }^{3}$ Department of Neurology, 463rd Hospital of PLA, Shenyang, China \\ *These authors contributed equally to this study. \\ Corresponding author: Z.Y. He \\ E-mail: zhiyihe4@gmail.com
}

Genet. Mol. Res. 13 (2): 3586-3598 (2014)

Received December 21, 2012

Accepted April 23, 2013

Published May 9, 2014

DOI http://dx.doi.org/10.4238/2014.May.9.2

\begin{abstract}
Ginsenoside Rh2 (Rh2) is a ginseng derivative used in Chinese traditional medicine. We investigated whether $\mathrm{Rh} 2$ can help prevent Alzheimer's disease symptoms and examined underlying mechanisms. We injected Rh2 into tg2576 Alzheimer's disease model mice and looked for behavioral improvement and senile plaque reduction in brain slices. We measured amyloid precursor protein (APP) metabolism species changes, amyloid beta40 and 42 levels and $\beta, \gamma$ secretase activity in primary hippocampal neurons. By living cell staining, we detected surface and endocytosed APP. We also measured cholesterol and lipid rafts in primary neurons. Rh2 treatment significantly improved learning and memory performance at 14 months of age; it also reduced brain senile plaques at this age. Based on in vitro experiments, we found that Rh2 treatment increased soluble APP $\alpha$ $(\mathrm{sAPP} \alpha)$ levels, increased CTF $\alpha / \beta$ ratios, and reduced amyloid beta 40
\end{abstract}


and 42 concentrations. Surface APP levels dramatically increased. Based on living cell staining, we found that Rh2 inhibited APP endocytosis. Based on lipid removal and reload experiments, we found that $\mathrm{Rh} 2$ can modulate APP by reducing cholesterol and lipid raft levels. We concluded that Rh2 improves learning and memory function in Alzheimer's disease model mice, and that this improvement is accomplished by reducing amyloid beta secretion and APP endocytosis, which in turn is achieved by reducing cholesterol and lipid raft concentrations.

Key words: Rh2; APP processing; Cholesterol; Lipid raft; Alzheimer's disease

\section{INTRODUCTION}

A key event in the pathogenesis of Alzheimer's disease (AD) is the proteolytic processing of the $\beta$-amyloid precursor protein (APP) by the two proteases $\beta$ - and $\gamma$-secretase, leading to the formation of the amyloid- $\beta$ (A $\beta$ ) peptide which accumulates in AD brains (Hardy, 2002). $\beta$-secretase (BACE1) cleavage of APP generates two products (Vassar et al., 1999): the $\mathrm{N}$-terminal secreted APP $\beta$ fragment (sAPP $\beta$ ) and the C-terminal membrane-bound fragment $(\mathrm{CTF} \beta / \mathrm{C} 99)$ which is further cleaved by $\gamma$-secretase complex (composed of presenilin $1 / 2$ or PS1/2, nicastrin, Aph1 and Pen2) to generate A $\beta$ peptides (Sun et al., 2012). In parallel with the amyloidogenic $\beta$-secretase pathway, APP can also be processed through the non-amyloidogenic $\alpha$-secretase pathway, which precludes the generation of A $\beta$. $\beta$-secretase cleavage of APPwt is thought to happen primarily within endosomal compartments (Cirrito et al., 2008), and cleavage of APP by $\alpha$-secretase has been suggested to occur mainly at the cell surface (Sisodia, 1992). Recent evidence increasingly implicates intracellular APP trafficking as the main mechanism regulating the access of APP to its secretase and thus A $\beta$ generation (Sannerud and Annaert, 2009).

There is growing evidence that cholesterol contributes to the pathogenesis of Alzheimer's disease. Elevated dietary cholesterol uptake increased amyloid plaque formation in rabbits and transgenic mice (Refolo et al., 2000), and cholesterol loading and depletion affects $\mathrm{A} \beta$ generation in cultured cells (Fassbender et al., 2001). In addition to the indirect effects of cholesterol on APP processing, recent findings suggest that cholesterol may directly modulate the activity of $\beta$-secretase (BACE1) (Kalvodova et al., 2005), while contradictory results have been reported for the effect of cholesterol on $\gamma$-secretase cleavage of APP (Wada et al., 2003). Additionally, it has been recently shown that increased cholesterol levels initiate endosome enlargement and increased amyloidogenic processing of APP (Cossec et al., 2010), supporting the notion that alterations in cholesterol metabolism could initiate $\mathrm{AD}$ pathogenesis.

Ginseng root has been commonly used in traditional Chinese medicine for over 2000 years as a tonic against stress (Lu et al., 2008). Although ginseng has potential adverse effects such as hypoglycemia and an increased risk of bleeding (Ang-Lee et al., 2001), the incidence of these adverse effects seems to be low (Topliss et al., 2002). Ginsenosides (steroidal glycosides), the major active components in the extracts of various kinds of ginseng, are responsible for the pharmacological effects of ginseng on the central nervous, cardiovascular, endocrine, and immune systems (Chen et al., 2006). There are two major classes of ginsenosides in ginseng, namely protopanaxatriols ( $\mathrm{Rg} 1, \mathrm{Rh} 2, \mathrm{Re}$, and $\mathrm{Rf}$ ) and protopanaxadiols ( $\mathrm{Rb} 1, \mathrm{Rb} 2, \mathrm{Rc}$, and Rd) (World Health Organization, 1999). Among them, ginsenoside Rh2 is one of the 
most active and abundant components and contributes to many of the effects of ginseng. Ginsenoside Rh2 (Rh2) is known to alleviate multi-drug resistance in cancer cells and attenuate amyloid-induced cytotoxicity (Shieh et al., 2008). Multi-drug resistance proteins and amyloid beta are predominantly localized in lipid rafts, the functions of these proteins are inhibited when lipid rafts are disrupted. On the basis of the above observations and considering its similar structure to cholesterol, it is tempting to speculate that Rh2 could disrupt lipid rafts (Urano et al., 2005). However, the exact molecular mechanism of whether and how Rh2 can protect $\mathrm{AD}$ occurrence is still unclear. Here, we investigated whether cholesterol and lipid rafts are involved in Rh2-regulated APP trafficking and processing.

\section{MATERIAL AND METHODS}

\section{Materials}

Ginsenosides were purchased from BTGin (Chung-Nam, Korea). Eagle's Minimum Essential Medium (DMEM) was purchased from American Type Culture Collection (ATCC, Rockville, MD, USA). Neurobasal A medium (phenol red-free), N2 supplement, fetal bovine serum, penicillin, streptomycin, and anti-APP antibodies (A8717; anti-APP C-terminus) were purchased from Sigma. anti-APP antibodies 6E10 and 22C11 (anti-APP N-terminus) were purchased from Signet and Chemicon, respectively.

\section{Primary neuron culture}

Cortex neurons were isolated from mouse embryos at day 16 of gestation. The cortex neurons were plated at densities of $20,000 \mathrm{cells} / \mathrm{cm}^{2}$ in $6-\mathrm{cm}$ tissue culture dishes containing poly-L-lysine ( $1 \mathrm{mg} / \mathrm{mL}$; Sigma Aldrich)-coated glass coverslips and Neurobasal medium supplemented with $2 \%$ B27 and $0.5 \mathrm{mM}$ glutamine (all from Invitrogen).

\section{Experimental animals and drug administration}

Forty tg2576 mice, 12 months old, and the same number of wild-type (WT) control mice of the same age were provided by the university animal facility. Before experiments, they were kept under defined environmental conditions $\left(25^{\circ} \mathrm{C}, 50 \%\right.$ relative humidity, 12 -h lightdark cycle) and were given standard diet and water ad libitum. Mice were divided into four groups: tg2576-vehicle; tg2576-Rh2; control-vehicle and control-Rh2 $(\mathrm{N}=20$ each group). Mice in both $\mathrm{Rh} 2$ and vehicle treatment groups were intraperitoneally injected with the related reagents in saline at a daily dose of $10 \mathrm{mg} / \mathrm{kg}$ body weight. After 8 weeks of Rh2 treatment, all mice were subjected to behavior experiments and then sacrificed for further experiments.

\section{Morris water maze test}

The Morris water maze (MWM) test was conducted according to Morris (1984). The water-filled $\left(23^{\circ} \mathrm{C}\right)$ black-colored tank $(150 \mathrm{~cm}$ diameter; $60 \mathrm{~cm}$ depth $)$ was arbitrarily divided into four quadrants of equal area. A circular platform $(10 \mathrm{~cm}$ in diameter) made of transparent Perspex was submerged $1.0 \mathrm{~cm}$ below the water surface with its center $37.5 \mathrm{~cm}$ from the pe- 
rimeter, in the middle of one quadrant (the target quadrant). A closed-circuit television camera was mounted onto the ceiling directly above the center of the pool to convey subject swimming trajectories and parameters to an electronic image analyzer.

\section{Procedure}

Habituation: The MWM test began with one-day habituation. The mice were forced to stay on the platform for $45 \mathrm{~s}$, which was $1.0 \mathrm{~cm}$ beneath the water. If the mice jumped down, they would be put back onto the platform, while the time record was re-started.

Hidden platform training and probe trial: Hidden platform training was performed according to Shieh et al. (2008) with variable interval probes to evaluate learning and memory repeatedly. The mice were subjected to one day habituation as described above. Starting on the following day, the hidden platform training was performed in two sessions a day, separated by $4 \mathrm{~h}$, for five consecutive days. The mice were placed in the pool facing the perimeter, allowed a maximal time of $90 \mathrm{~s}$ to find the platform and step onto it. If the mice crossed the platform without stopping (jumping immediately into the water), it was left to swim on. After finding the platform, the mice were allowed to stay on it for $15 \mathrm{~s}$. If the mice failed to find the platform in the allotted time, it was guided onto the platform and allowed to stay on it for $15 \mathrm{~s}$. After finishing all three trials, the mice were taken from the platform, gently dried with a towel and returned to their home cage. Four hours later, the next training session started. Between two successive trials, the water was stirred to erase olfactory traces of the previous swimming pattern. The platform was left in the same location throughout the hidden platform training. The same experiment would last for 7 days in a row.

\section{A $\beta$ levels}

$A \beta$ levels in the culture medium of primary neurons and in the hippocampus tissue of the tg2576 mice were measured using the Mouse/Rat A $\beta$ (1-42) ELISA kit (IBL International $\mathrm{GmbH}$, Hamburg, Germany) according to manufacturer protocol. Before measurement of $A \beta$ release into the culture medium, the medium was replaced by fresh serum-free DMEM. After cells were treated as described above, the medium was collected and $A \beta$ levels were measured by A $\beta$ ELISA kit using the Victor2 Multilabel Counter (Perkin Elmer/Wallace) at a wavelength of $450 \mathrm{~nm}$. A $\beta$ levels were measured in hippocampal tissue that was homogenized and centrifuged $\left(70,000 \mathrm{~g}\right.$ for $20 \mathrm{~min}$ at $4^{\circ} \mathrm{C}$ ), using a $100-\mu \mathrm{L}$ sample of the supernatant. Spectrophotometric data were then obtained using the same Victor 2 Multilabel Counter at $450 \mathrm{~nm}$. The protein concentration was measured as described above. $A \beta$ levels were corrected for protein content.

\section{$\alpha$ - and $\beta$-secretase activity}

$\alpha$ - and $\beta$-secretase activity was measured following manufacturer instructions (GBD, San Diego, CA, USA). The protein concentration was determined, and equal amounts of protein and primary antibody were added to each well for one hour in a $37^{\circ} \mathrm{C}$ incubator. The wells were then washed 5 times and secondary antibody was added; the plate was then placed in a $22^{\circ} \mathrm{C}$ incubator for another $20 \mathrm{~min}$. Stop buffer was added and the final value was determined at $450 \mathrm{~nm}$ using a spectrophotometric microplate reader. 


\section{Cell surface biotinylation}

Cell surface biotinylation was performed using EZ-Link ${ }^{\mathrm{TM}}$ Sulfo-NHS-SS-Biotin (1 $\mathrm{mg} / \mathrm{mL}$ ) and Neutravidin ${ }^{\mathrm{TM}}$ Protein beads (all from Pierce) as described (Sharifov et al., 2011). During the biotinylation procedure, all reagents and cell cultures were kept on ice. The cells were washed three times in PBS, pH 8.5 (measured at $4^{\circ} \mathrm{C}$ ), then incubated in $1 \mathrm{mg} / \mathrm{mL}$ EZLink $^{\mathrm{TM}}$ Sulfo-NHS-SS-Biotin solution (Pierce) in PBS, $\mathrm{pH}$ 8.5, for 30 min and washed twice with $100 \mathrm{mM}$ glycine in PBS on plates. The cells were then harvested in lysis buffer (1\% NP40, $0.1 \%$ SDS in PBS, $\mathrm{pH} 8.5$ ) in the presence of a protease inhibitor cocktail (Roche Applied Science, Germany). Lysates were incubated for $20 \mathrm{~min}$ on ice, centrifuged at $4^{\circ} \mathrm{C}$ for $10 \mathrm{~min}$ at $16,000 \mathrm{~g}$. Supernatants (normalized per mg protein) were incubated overnight with $50 \mu \mathrm{L}$ Neutravidin ${ }^{\mathrm{TM}}$ Protein beads (Pierce) with constant rocking. Neutravidin beads were washed three times with PBS, then dried by aspiration, resuspended in $2 \mathrm{X}$ sample buffer, heated for 10 min at $70^{\circ} \mathrm{C}$ and used for Western blot analysis of cell surface APP. For determination of total APP levels, the cell lysates were blotted and analyzed as indicated. The signals were quantified and expressed as the ratio of the cell surface and total (mature+immature) APP levels.

\section{Measurement of cholesterol content}

Cells grown in 60-mm dishes were washed three times with PBS at room temperature. Ice-cold $1 \mathrm{X}$ cholesterol measurement reaction buffer $(0.05 \mathrm{M} \mathrm{NaCl}, 5 \mathrm{mM}$ cholic acid, $0.1 \%$ Triton $\mathrm{X}-100,0.1 \mathrm{M}$ potassium phosphate, $\mathrm{pH}$ 7.4) was added to the dishes and cells were lysed for $20 \mathrm{~min}$ at $4^{\circ} \mathrm{C}$. Lysates were transferred to $1.5 \mathrm{-mL}$ Eppendorf tubes and sonicated briefly. Protein concentration was measured using the Bradford Ultra kit (Novexin Ltd., Cambridge, UK), and cholesterol was measured using the Amplex Red Cholesterol Assay kit (Molecular Probes, Inc., Eugene, OR, USA).

\section{Cholesterol depletion and reloading}

To load cholesterol, primary neurons were treated with $100 \mu \mathrm{M}$ M $\beta C D$-cholesterol complex (Sigma Aldrich) for $4 \mathrm{~h}$ after $24 \mathrm{~h}$ incubation in 10\% LPDS medium. For chronic cholesterol depletion, we cultured neurons in 10\% lipid-deficient serum (LPDS, Cocalico Biologicals Inc.) medium plus methyl- $\beta$-cyclodextrin (M $\beta C D$, Sigma Aldrich) for $24 \mathrm{~h}$.

\section{Vital staining}

Immunocytochemistry was performed on mice cortex neurons as follows. Cortex neurons were directly incubated with primary antibody in incubator for $30 \mathrm{~min}$ and washed with cold PBS three times. The coverslips were put back in normal NB27 medium for another 30 min to allow endocytosis. Afterwards, cells were fixed with $4 \%$ paraformaldehyde for $15 \mathrm{~min}$ and directly incubated with Alexa Fluor 488 or 594-conjugated secondary antibody for $1 \mathrm{~h}$. The cells were washed three times with cold PBS and then blocked with non-fluorescenceconjugated secondary antibody in the same species overnight. After permeabilization with $0.1 \%$ Triton X-100 for $5 \mathrm{~min}$, the neurons were incubated with another kind of secondary antibody for staining endocytosis. 
For lipid raft staining, living cells were stained directly by Alexa Fluor-594 conjugated with cholera toxin subunit B (CTx-B) for $30 \mathrm{~min}$ in an incubator, and then observed under a microscope with or without mounting.

Confocal images were acquired on an inverted laser scanning confocal microscope (Zeiss Axiovert 200M) with a 60X/1.3 N.A. water immersion lens. Images were taken and analyzed with the LSM 510 confocal software (Zeiss).

\section{Immunohistochemistry}

After 6 min of perfusion with saline, mouse brains were divided sagittally in half; one half was immersed in paraformaldehyde $(4 \%)$ for $26 \mathrm{~h}$. Serial vibratome sections (20 $\mu \mathrm{m}$, two sections from each mouse) were incubated with a mixture of the two media: (A) $5 \mathrm{mg}$ acetylthiocholine iodide, $6.5 \mathrm{~mL} 0.1 \mathrm{mM}$ acetate buffer, $\mathrm{pH}$ 6.0, $0.5 \mathrm{~mL} 30 \mathrm{mM}$ copper sulfate, $1.0 \mathrm{~mL}$ distilled water, and $0.2 \mathrm{~mL} 4 \mathrm{mM}$ iso-octamethyl pyrophosphoramide (iso-OMPA); and (B) $1.0 \mathrm{~mL} 5 \mathrm{mM}$ potassium ferricyanide at $37^{\circ} \mathrm{C}$ for $5 \mathrm{~min}$ and rinse with tap water for $10 \mathrm{~s}$. Afterwards, they were developed with freshly prepared 3-3' diamino benzidine tetrahydrochloride ( $25 \mathrm{mg}$ in $100 \mathrm{~mL} 0.05 \mathrm{M}$ phosphate buffer, $\mathrm{pH} 7.0)$, containing $0.015 \% \mathrm{H}_{2} \mathrm{O}_{2}$ for $5 \mathrm{~min}$ at room temperature, and then washed with tap water, dehydrated and mounted. Sections were reacted with anti-A $\beta$ antibody 3D6 (dilution 1:1000) to identify $\mathrm{A} \beta$ deposits. Areas with $\mathrm{A} \beta$ plaques were analyzed using the MetaMorph software evaluating multiple sections of the same level in each animal group. To ensure objective assessments and reliability of the results, brain sections from mice in any given experiment were blind coded and processed in parallel. Codes were revealed only after the analysis was completed.

\section{Statistical analysis}

Statistical analysis was done by the SPSS 15.0 software (Statistical Package for the Social Sciences). All data were submitted to the Kolmogorov-Smirnov test for normal distribution and Levene's test of equal variances for variance homogeneity. In some cases, square root transformation was employed for correction for heterogeneity of variance. For two-variable comparisons, a two independent samples $t$-test was used. For multiple variable comparisons, data were analyzed by one-way analysis of variance (ANOVA) followed by pair wise $t$-tests.

\section{RESULTS}

\section{$\mathrm{Rh} 2$ treatment improves the behavior performance of $\operatorname{tg} 2576$ transgenic mice}

To investigate whether long-term $\mathrm{Rh} 2$ treatment improved the ability of learning and memory, we tested the spatial learning and memory function of Rh2-treated tg2576 mice by the MWM. The spatial learning ability of mice was indicated by the path length to find the platform. In this study, tg2576 mice and WT mice were treated with vehicle or Rh2 for 8 weeks separately, and results showed that while $\mathrm{Rh} 2$ treatment appeared to have little influence on WT mice, it significantly decreased the path length of tg2576 mice $(\mathrm{P}<0.01$, Figure 1a). These data suggested that Rh2 treatment could specifically improve spatial learning ability of $\operatorname{tg} 2576$ mice but not WT mice. 

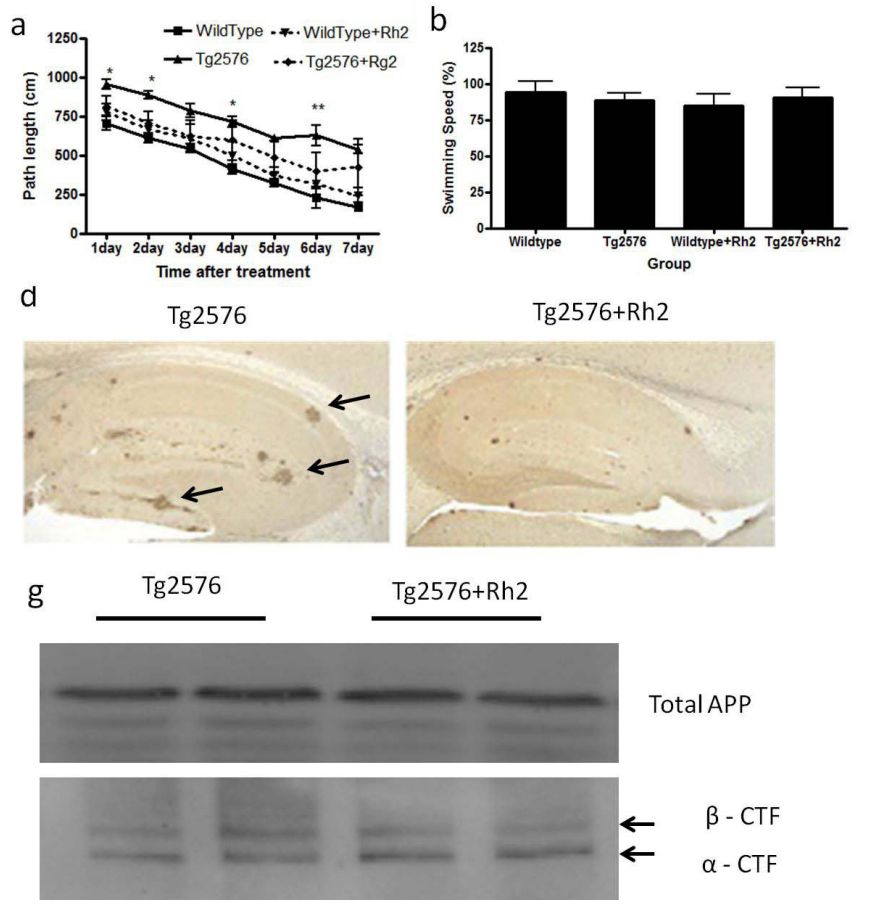

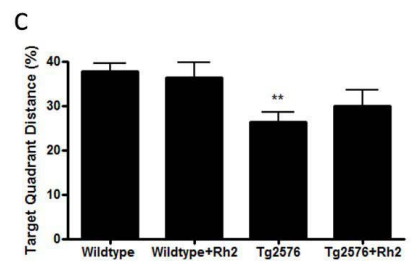

e
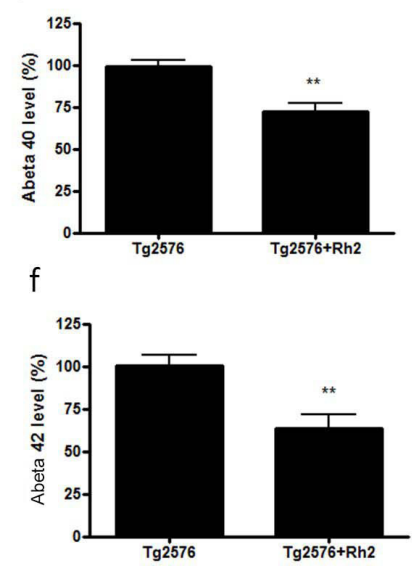

Figure 1. Rh2 treatment could improve the memory performance of tg2576 mice and reduce Abeta plaques: $\mathrm{Rh} 2$ or vehicle treated mice (12-month old) were treated with $10 \mathrm{mg} / \mathrm{kg} \mathrm{Rh} 2$ or vehicle for 8 weeks. After that spatial reference memory was tested in Morris water maze task. The performance of rats in the task was recorded as the path length to find the platform. The path length to the submerged platform across trial days were plotted as line charts (a), and the swimming speed and existence in target quadrant was shown in b and c separately. After behavior test, the mice were scarified and brain samples were collected. Abeta plaque number (d), abeta 40 and 42 (e,f) and APP metabolism species $(\mathrm{g})$ were measured. Values are presented as Mean $\pm \mathrm{SD}$ of four determinations. $* \mathrm{P}<0.05$, $* * \mathrm{P}<0.01$ versus corresponding control group.

To test whether the swim speed confounded the effects of $\mathrm{Rh} 2$ on the path length to find the platform, we also measured the swim speed of mice in this study. However, no significant differences in swim speed were found between the different groups (Figure 1b), suggesting that the swim speed did not confound the path length as measurement of the spatial learning ability in this study.

After escape training, the spatial memory of animals was also assessed by a spatial bias test. The data are reported as the percentage of swimming distance in the target quadrant in the absence of the submerged platform. In this study, the swimming distance in the target quadrant of vehicle- and Rh2-treated tg2576 mice were $25.21 \pm 7.22$ and $30.70 \pm 5.85 \%$, respectively, while WT mice did not show a great difference (Figure 1c). These data suggested the beneficial effect of Rh2 on the spatial memory of $\operatorname{tg} 2576$ mice.

\section{Rh2 treatment promotes non-amyloid cleavage of APP in the hippocampus of $\operatorname{tg} 2576$ mice}

As we already found, $\mathrm{Rh} 2$ treatment could specifically improve learning and memory 
of tg2576 mice. We next wondered whether this improvement was related to attenuated Abeta accumulation. Here, we found by immunohistochemistry staining that Abeta plaques in Rh2treated mouse brain were significantly reduced compared to control tg2576 mice (Figure 1d), while ELISA measurement also found that in mouse hippocampus lysate, the amounts of both Abeta 40 and 42 were decreased in the Rh 2 treatment group (Figure 1e, f). Western blot results showed that while total amount of APP was barely changed, the $\alpha / \beta-C T F$ ratio was increased in the $\mathrm{Rh} 2$ treatment group compared with control tg2576 mice (Figure 1g).

\section{Rh2 changes APP trafficking and processing in tg2576 primary neuron culture}

The levels of secreted $A \beta$ 1-40 and $A \beta$ 1-42 in the conditioned medium of $\operatorname{tg} 2576$ primary cortex neurons were increased in a time-dependent manner, with a relatively stable level at DIV (days in vitro) 5 for A $\beta$ 1-42 and DIV 4 for A $\beta 1-40$ (data not shown), respectively. The effect of ginsenoside $\mathrm{Rh} 2$ on the secreted $A \beta$ 1-40 and $A \beta$ 1-42 was then examined in the conditioned medium of primary neurons at DIV 7. As shown in Figure 2, Rh2 treatment significantly decreased the extracellular levels of $A \beta 1-40$ and $A \beta 1-42$ in a time-dependent manner, particularly at a dose of $2.5 \mu \mathrm{M}$ for $12 \mathrm{~h}$.

a

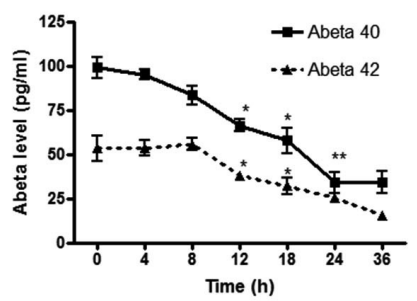

$\mathrm{C}$

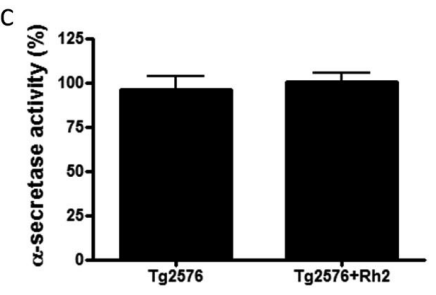

e

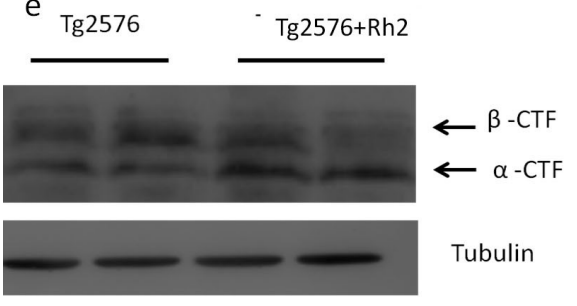

b

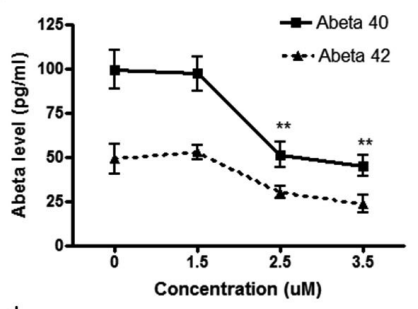

$\mathrm{d}$

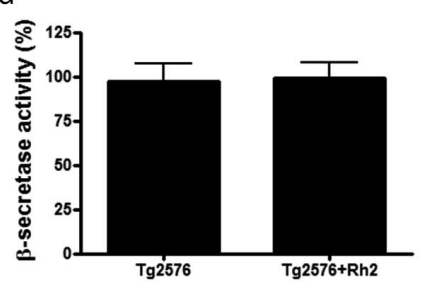

$f$

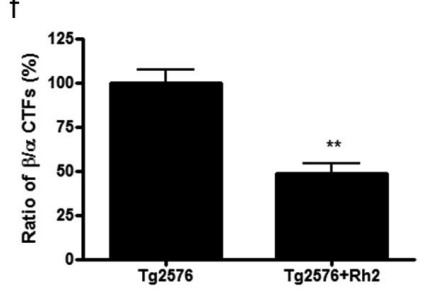

Figure 2. $\beta$-cleavage in Rh2 treated primary neuron were reduced: Primary cortex neuron from mice embryo were cultured on cover-slip, and abeta level was measured after treatment with different concentration of Rh2 for different time start at DIV 7 (a,b). Although both a and b secretase activity did not change a lot (c,d) after Rh2 treatment, the ratio of $\beta$-and $\alpha$-CTF was remarkably decreased after Rh2 treatment $(\mathrm{e}, \mathrm{f})$. Values are reported as means $\pm \mathrm{SD}$ of four determinations. ${ }^{*} \mathrm{P}<0.05, \mathrm{P}<0.01$ versus corresponding control group. 
The activities of the $\alpha$ - and $\beta$-cleavage event were executed through $\alpha$-CTF and $\beta$-CTF levels and $\alpha$ - and $\beta$-secretase activity. As shown in Figure 2e, there was a higher protein level of $\beta$-CTF in tg2576 mouse primary neurons, when compared with the Rh2 treatment group. In contrast, a lower protein level of $\alpha$-CTF was shown in the non-treatment group. Meanwhile, we found that the activity of $\alpha$ - and $\beta$-secretase did not change (Figure 2c, d). These results indicated that ginsenoside Rh2 may increase the $\alpha$-cleavage event indirectly without altering its activity.

Since the sub-cellular location of APP would also influence its processing, we next determined the surface distribution of APP in primary neurons with and without $\mathrm{Rh} 2$ treatment by biotin conjugation. The results in Figure 3a clearly showed that surface APP and soluble APP $\alpha(\mathrm{sAPP} \alpha)$ were increased after Rh2 treatment compared with control group, while total APP amount was not changed much. This result indicated that Rh2 treatment could change APP distribution. We further employed vital staining to detect APP endocytosis. The results in Figure $3 \mathrm{~b}$ and $\mathrm{c}$ showed that compared with control group, surface APP was increased after $\mathrm{Rh} 2$ treatment (green channel) and that endocytosis of APP was decreased (far-red channel, cy5), while total APP did not change much (red channel). All of these results suggested that Rh2 treatment could inhibit APP endocytosis, increase APP cell membrane distribution, and finally decrease Abeta secretion and increase sAPP $\alpha$ secretion.

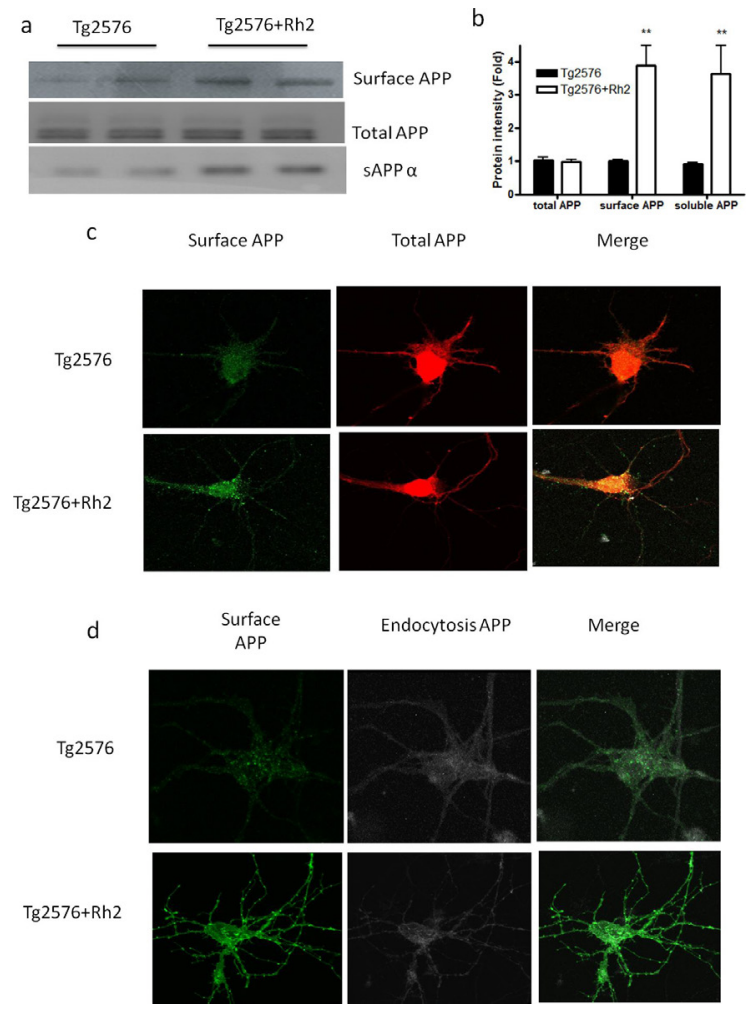

Figure 3. Surface APP endocytosis was inhibited after Rh2 tretment: After Rh2 treatment, although total amount of APP was kept the same, the surface APP and soluble APP were both increased significantly $(\mathrm{a}, \mathrm{b})$. The living staining results showed while surface APP increased, the endocytosis APP was decreased after Rh2 treatments (c,d). Values are reported as means $\pm \mathrm{SD}$ of four determinations. ${ }^{*} \mathrm{P}<0.05, \mathrm{P}<0.01$ versus corresponding control group. 


\section{Rh2 reduces cholesterol and lipid raft levels in primary neurons while cholesterol overload can restore $\mathbf{R h} 2$-reduced Abeta level}

Since lipid raft is important for APP endocytosis, we wondered whether Rh2 effects on APP distribution influence cholesterol levels. By measuring the cholesterol levels of primary neurons with or without $\mathrm{Rh} 2$ treatment, we found that $\mathrm{Rh} 2$ significantly decreased cholesterol level in primary neurons (Figure 4a). Afterwards, we also stained primary neurons with lipid raft marker CTx-B (red channel) and found that the membrane distribution of CTX-B was significantly reduced after Rh2 treatment, which indicated that Rh2 may lower the Abeta level by decreasing the amount of lipid rafts (Figure $4 b$ ).
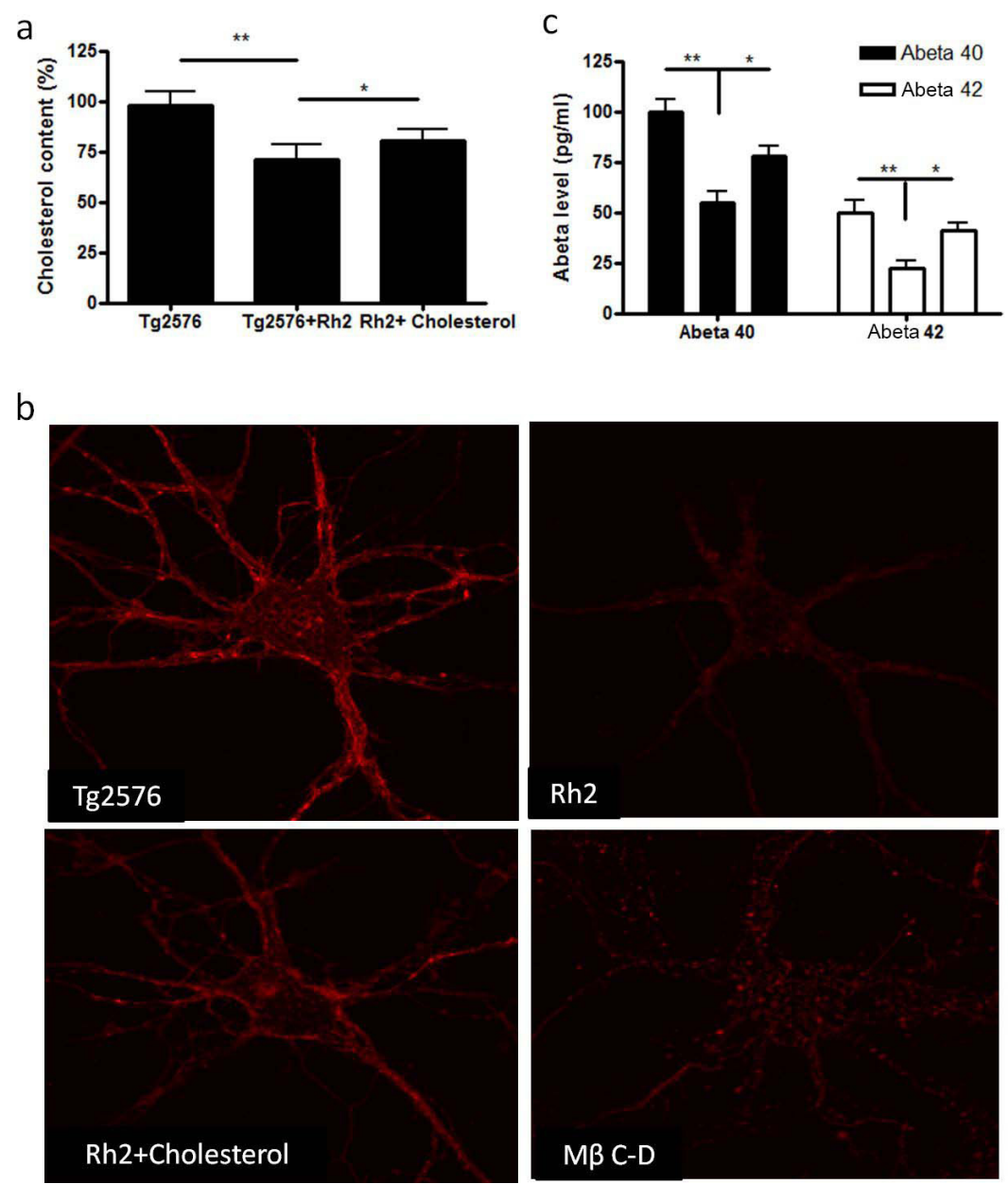

Figure 4. Rh2 effects on reducing Abeta was related to cholesterol and lipid raft: After Rh2 treatments, the cholesterol content of primary neuron was reduced compare with non-treated cell, while after cholesterol re-loading, both cholesterol and abeta level was increased again $(\mathrm{a}, \mathrm{c})$. CTx-B staining results show after Rh2 treatment, the lipid raft intensity was reduced (b). Values are reported as means $\pm \mathrm{SD}$ of four determinations. $* \mathrm{P}<0.05, \mathrm{P}<0.01$ versus corresponding control group. 
Finally, we administered high-cholesterol containing serum treated neuron combined with Rh2 treatment and found that, along with recovery of the cellular cholesterol level, the amount of Abeta 40/42 was also recovered (Figure 4c). These results strongly indicated that $\mathrm{Rh} 2$ treatment could decrease Abeta secretion by decreasing cellular cholesterol and lipid raft levels.

\section{DISCUSSION}

In the present study, we established that Rh2 treatment protects against $\mathrm{A} \beta$-mediated abnormalities in tg2576, a well-characterized AD mouse model. Rh2 treatment reversed behavior defects in tg2576 mice, and reduced the number of senile plaques in brain slices. First, we demonstrated a role of $\mathrm{Rh}-2$ in the regulation of APP endocytosis, a potential mechanism of Rh2-mediated reduction in $\mathrm{A} \beta$ accumulation in brain, both in primary neuronal culture and in $\operatorname{tg} 2576$ mice in vivo. Second, we showed that the inhibition of APP endocytosis was mediated by reduced lipid raft level. Third, we found $\mathrm{Rh} 2$ treatment could improve learning and memory function of $\operatorname{tg} 2576$ mice. These results provide additional evidence of the protective effects of Rh2 on A $\beta$-induced amyloid pathology and impaired cognitive function, which will significantly strengthen and support the potential of $\mathrm{Rh} 2$ for clinical translation in the future.

The use of ginseng for improving cognitive performance has become increasingly popular in recent years (Li et al., 2011; Zhang et al., 2012). Particularly, ginsenoside $\mathrm{Rh} 2$ was recently found to protect neurons from hypoxia-induced damage, indicating that Rh2 could exert a neuron-protective effect through some mechanisms (Shuangyan et al., 2012). The present study employed APP-overexpressing tg2576 mice to investigate the effect of Rh2 on A $\beta$ generation in vivo and found that $\mathrm{Rh} 2$ could actually reduce the Abeta level in mouse brain and primary neurons. As numerous works have found, Abeta generation was involved in $\alpha-, \beta$ - and $\gamma$-secretase complex activity. To further determine whether Rh2-induced decrease in Abeta was involved in this secretase complex, we directly measured those secretase activities and found that there were no significant changes in any of these secretase activities. Furthermore, as total APP level did not change considerably, the decrease in Abeta may have been caused by altered APP distribution.

To confirm this hypothesis, we determined cell surface APP and found that $\mathrm{Rh} 2$ treatment significantly increased APP surface distribution, which would further facilitate the activation of the non-amyloid cleavage pathway. The increased cell membrane distribution may have been caused by an increased insertion event or reduced endocytosis process. We further employed vital staining to investigate this question. By briefly staining the N-terminal of APP and then allowing endocytosis for a little while, we found that after Rh2 treatment, there was an increase in surface APP level and a dramatic decrease in endocytosed APP. This result strongly indicated that Rh2 could inhibit APP endocytosis and thus increase membrane APP retention.

Cholesterol levels have been implicated in the pathogenesis of Alzheimer's disease (AD) (Sharifov et al., 2011; Reiss and Voloshyna, 2012) with disturbed cholesterol homeostasis, showing neurodegeneration, tau pathology and increased formation of $A \beta$ (Yamazaki et al., 2001; Burns et al., 2003; Jin et al., 2004). It was previously reported that APP is endocytosed via the clathrin-dependent pathway (Schneider et al., 2008), while many previous works also found that lipid rafts could have a function in APP endocytosis and Abeta generation. Although lipid raft-associated proteins are known to be generally internalized through clathrin-independent pathways, recent evidence suggests a link between lipid rafts and clathrin- 
mediated endocytosis. Indeed, cholera and anthrax toxins enter the cells through lipid rafts by a clathrin-dependent mechanism (Midha and Bhatnagar, 2009; Auriac et al., 2010). In addition, lipid raft-associated proteins (such as CD317 or the B-cell antigen receptor) can be internalized in clathrin-coated pits (Stoddart et al., 2002; Rollason et al., 2007). APP has been proposed to interact directly with cholesterol (Beel et al., 2008), and a recent study found that the C-terminal of APP contains the cholesterol-binding domain (Barrett et al., 2012), which further confirmed the notion that lipid raft distribution of APP may accelerate Abeta pathology.

Because Rh2 is structurally similar to cholesterol and can interact with membrane lipids (Yue et al., 2007), it is possible that Rh2 exerts its effect by modulating cholesterol levels and rafts, thereby altering lipid raft function. The present study found that Rh2 treatment could reduce the cellular cholesterol level and thus disrupt lipid raft distribution. To further confirm whether Rh2-induced lipid raft perturbation would inhibit Abeta generation, we carried out cholesterol reloading experiments. The results showed that after Rh2 treatment, cells supplied with high-cholesterol containing medium had recovered Abeta generation to almost normal levels, indicating that $\mathrm{Rh} 2$ induced a decrease in Abeta mainly by reducing cholesterol levels.

In summary, our results clearly demonstrated the protective effect of $\mathrm{Rh} 2$ treatment against cerebral $\mathrm{A} \beta$ accumulation, neuropathological change, and impaired learning/memory in a transgenic mouse model for AD. Notably, $\mathrm{Rh} 2$ treatment is able to reverse substantially $\mathrm{A} \beta$ induced deteriorating effects observed in an aged transgenic mouse model for $\mathrm{AD}$, which exhibits extensive amyloid pathology and cognitive decline. The mechanism through which Rh2 regulates APP processing involves reducing the cellular cholesterol level and thereby inhibiting the membrane APP endocytosis process. Together with previous studies, our data indicate that $\mathrm{Rh} 2$ may be a potential therapeutic agent for preventing and halting AD progression.

\section{REFERENCES}

Ang-Lee MK, Moss J and Yuan CS (2001). Herbal medicines and perioperative care. JAMA 286: 208-216.

Auriac A, Willemetz A and Canonne-Hergaux F (2010). Lipid raft-dependent endocytosis: a new route for hepcidinmediated regulation of ferroportin in macrophages. Haematologica 95: 1269-1277.

Barrett PJ, Song Y, Van Horn WD, Hustedt EJ, et al. (2012). The amyloid precursor protein has a flexible transmembrane domain and binds cholesterol. Science 336: 1168-1171.

Beel AJ, Mobley CK, Kim HJ, Tian F, et al. (2008). Structural studies of the transmembrane C-terminal domain of the amyloid precursor protein (APP): does APP function as a cholesterol sensor? Biochemistry 47: 9428-9446.

Burns M, Gaynor K, Olm V, Mercken M, et al. (2003). Presenilin redistribution associated with aberrant cholesterol transport enhances $\beta$-amyloid production in vivo. J. Neurosci. 23: 5645-5649.

Chen F, Eckman EA and Eckman CB (2006). Reductions in levels of the Alzheimer's amyloid $\beta$ peptide after oral administration of ginsenosides. FASEB J. 20: 1269-1271.

Cirrito JR, Kang JE, Lee J, Stewart FR, et al. (2008). Endocytosis is required for synaptic activity-dependent release of amyloid- $\beta$ in vivo. Neuron 58: 42-51.

Cossec JC, Simon A, Marquer C, Moldrich RX, et al. (2010). Clathrin-dependent APP endocytosis and A $\beta$ secretion are highly sensitive to the level of plasma membrane cholesterol. Biochim. Biophys. Acta 1801: 846-852.

Fassbender K, Simons M, Bergmann C, Stroick M, et al. (2001). Simvastatin strongly reduces levels of Alzheimer's disease $\beta$-amyloid peptides A $\beta 42$ and A $\beta 40$ in vitro and in vivo. Proc. Natl. Acad. Sci. U. S. A. 98: 5856-5861.

Hardy J (2002). Testing times for the "amyloid cascade hypothesis". Neurobiol. Aging 23: 1073-1074.

Jin LW, Shie FS, Maezawa I, Vincent I, et al. (2004). Intracellular accumulation of amyloidogenic fragments of amyloid- $\beta$ precursor protein in neurons with Niemann-Pick type $\mathrm{C}$ defects is associated with endosomal abnormalities. Am. $J$. Pathol. 164: 975-985.

Kalvodova L, Kahya N, Schwille P, Ehehalt R, et al. (2005). Lipids as modulators of proteolytic activity of BACE: involvement of cholesterol, glycosphingolipids, and anionic phospholipids in vitro. J. Biol. Chem. 280: 36815-36823. 
Li WZ, Li WP, Zhang W, Yin YY, et al. (2011). Protective effect of extract of Astragalus on learning and memory impairments and neurons' apoptosis induced by glucocorticoids in 12-month-old male mice. Anat. Rec. (Hoboken.) 294: 1003-1014.

Lu XZ, Wang JH, Wu X, Zhou L, et al. (2008). Ginsenoside Rg1 promotes bone marrow stromal cells proliferation via the activation of the estrogen receptor-mediated signaling pathway. Acta Pharmacol. Sin. 29: 1209-1214.

Midha S and Bhatnagar R (2009). Genetic immunization with GPI-anchored anthrax protective antigen raises combined CD1d- and MHC II-restricted antibody responses by natural killer T cell-mediated help. Vaccine 27: 1700-1709.

Morris R (1984). Developments of a water-maze procedure for studying spatial learning in the rat. J. Neurosci. Methods 11: 47-60.

Refolo LM, Malester B, LaFrancois J, Bryant-Thomas T, et al. (2000). Hypercholesterolemia accelerates the Alzheimer's amyloid pathology in a transgenic mouse model. Neurobiol. Dis. 7: 321-331.

Reiss AB and Voloshyna I (2012). Regulation of cerebral cholesterol metabolism in Alzheimer disease. J. Investig. Med. 60: $576-582$

Rollason R, Korolchuk V, Hamilton C, Schu P, et al. (2007). Clathrin-mediated endocytosis of a lipid-raft-associated protein is mediated through a dual tyrosine motif. J. Cell Sci. 120: 3850-3858.

Sannerud R and Annaert W (2009). Trafficking, a key player in regulated intramembrane proteolysis. Semin. Cell Dev. Biol. 20: 183-190.

Schneider A, Rajendran L, Honsho M, Gralle M, et al. (2008). Flotillin-dependent clustering of the amyloid precursor protein regulates its endocytosis and amyloidogenic processing in neurons. J. Neurosci. 28: 2874-2882.

Sharifov OF, Nayyar G, Garber DW, Handattu SP, et al. (2011). Apolipoprotein E mimetics and cholesterol-lowering properties. Am. J. Cardiovasc. Drugs 11: 371-381.

Shieh PC, Tsao CW, Li JS, Wu HT, et al. (2008). Role of pituitary adenylate cyclase-activating polypeptide (PACAP) in the action of ginsenoside Rh2 against $\beta$-amyloid-induced inhibition of rat brain astrocytes. Neurosci. Lett. 434: 1-5.

Shuangyan W, Ruowu S, Hongli N, Bei Z, et al. (2012). Protective effects of Rg2 on hypoxia-induced neuronal damage in hippocampal neurons. Artif. Cells Blood Substit. Immobil. Biotechnol. 40: 142-145.

Sisodia SS (1992). $\beta$-amyloid precursor protein cleavage by a membrane-bound protease. Proc. Natl. Acad. Sci. U. S. A. 89: 6075-6079.

Stoddart A, Dykstra ML, Brown BK, Song W, et al. (2002). Lipid rafts unite signaling cascades with clathrin to regulate BCR internalization. Immunity 17: 451-462.

Sun M, Zhou T, Zhou L, Chen Q, et al. (2012). Formononetin protects neurons against hypoxia-induced cytotoxicity through upregulation of ADAM10 and sA $\beta P P \alpha$. J. Alzheimers Dis. 28: 795-808.

Topliss JG, Clark AM, Ernst E and Hufford CD (2002). Natural and synthetic substances related to human health. Pure Appl. Chem. 74: 1957-1985.

Urano Y, Hayashi I, Isoo N, Reid PC, et al. (2005). Association of active gamma-secretase complex with lipid rafts. $J$. Lipid. Res. 46: 904-912.

Vassar R, Bennett BD, Babu-Khan S, Kahn S, et al. (1999). $\beta$-secretase cleavage of Alzheimer's amyloid precursor protein by the transmembrane aspartic protease BACE. Science 286: 735-741.

Wada S, Morishima-Kawashima M, Qi Y, Misono H, et al. (2003). Gamma-secretase activity is present in rafts but is not cholesterol-dependent. Biochemistry 42: 13977-13986.

World Health Organization (1999). WHO Monographs on Selected Medicinal Plants. Geneva: World Health Organization, 168-183.

Yamazaki T, Chang TY, Haass C and Ihara Y (2001). Accumulation and aggregation of amyloid $\beta$-protein in late endosomes of Niemann-pick type C cells. J. Biol. Chem. 276: 4454-4460.

Yue PY, Mak NK, Cheng YK, Leung KW, et al. (2007). Pharmacogenomics and the Yin/Yang actions of ginseng: antitumor, angiomodulating and steroid-like activities of ginsenosides. Chin. Med. 2: 6.

Zhang X, Wang J, Xing Y, Gong L, et al. (2012). Effects of ginsenoside Rg1 or 17ß-estradiol on a cognitively impaired, ovariectomized rat model of Alzheimer's disease. Neuroscience 220: 191-200. 